\title{
Generation of Selective Fringes With Cascaded Long-Period Gratings
}

\author{
Ignacio Del Villar, M. Achaerandio, Francisco J. Arregui, Member, IEEE, and \\ Ignacio. R. Matias, Senior Member, IEEE
}

\begin{abstract}
The inscription of cascaded long-period gratings of different periods in optical fiber permits us to obtain both narrowband and wideband filters in the same spectrum. If used as an optical transducer, it may permit us to detect with the same device-wide and small parameter changes. The transmission spectra have been simulated using a theoretical model based on LP mode coupling and have been also experimentally demonstrated.
\end{abstract}

Index Terms-Coupled-mode analysis, long-period gratings, Mach-Zehnder interferometer, optical fiber sensors.

\section{INTRODUCTION}

D URING THE last decade, an increasing amount of effort has been devoted to the analysis and fabrication of longperiod fiber gratings (LPFGs) [1]-[5]. These devices have a period typically higher than $100 \mu \mathrm{m}$ [5]. Consequently, there is a coupling between the core mode and the copropagating cladding modes, unlike in fiber Bragg gratings (FBGs). This property permits the development of interesting applications in optical communications and sensor fields [1], [5]. The modal coupling induces some attenuation bands in the transmission expression. Though the simple phase matching condition is sufficient to give an approximation of the central wavelength of the attenuation band, the modified phase matching condition is often preferred [4]

$$
\beta_{01}(\lambda)+s_{0} \zeta_{01,01}(\lambda)-\left(\beta_{0 j}(\lambda)+s_{0} \zeta_{0 j, 0 j}(\lambda)\right)=\frac{2 \pi}{\Lambda N}
$$

where $\beta_{01}$ and $\beta_{0 j}$ are the propagation constants of the core and the $j$ cladding modes, respectively, $\varsigma_{01,01}$ and $\varsigma_{0 j, 0 j}$ are the self-coupling coefficients of the core and the $j$ cladding modes, $s_{0}$ is the coefficient of the dc Fourier component of the grating, $\Lambda$ is the period of the grating, and $N$ is the diffraction order.

On the other hand, the depth of the attenuation band depends on the length of the grating $L$ and the cross-coupling coefficient $k_{i}$ between the core and the cladding mode [5]

$$
T_{i}=\cos ^{2}\left(k_{i} L\right)
$$

Manuscript received February 8, 2006; revised March 9, 2006. This work was supported in part by the Spanish Ministerio de Ciencia y Tecnologia and FEDER under Grant CICYT-TIC 2004-05936-C02-01/MIC, by Gobierno de Navarra, and by FPU MECD.

The authors are with the Electrical and Electronic Engineering Department, Public University of Navarra, 31006 Pamplona, Spain (e-mail: ignacio.delvillar@unavarra.es; acha@unavarra.es; parregui@unavarra.es; natxo@unavarra.es).

Digital Object Identifier 10.1109/LPT.2006.877567
One of the main problems of LPFGs is that they show wide attenuation bands. If LPFGs are used as optical sensors, this fact reduces the resolution of the measurements performed. The fabrication of a Mach-Zehnder interferometer by cascading two LPFGs of attenuation band of depth $3 \mathrm{~dB}$ [6]-[8] solves this problem. All the attenuation bands in the transmission spectrum will present interference fringes of narrower width than the original attenuation band. This idea has been applied for sensing purposes with the problem that the wavelength shift of the fringes generated has a limited path [8]. Here, we propose, for the first time to our knowledge, a device where attenuation bands with and without fringes are generated in the same transmission spectrum. In this way, wider wavelength shifts with lower resolution can be measured using the attenuation band without fringes and shorter wavelength shifts with higher resolution can be also measured using the attenuation band with fringes. In the next section, the design is presented and some experimental results corroborate the theoretical predictions.

\section{CASCADEd LPFGs with Single ANd Double Period}

The device in Fig. 1 consists of two LPFGs separated by a gap. The key point is that the period of the second one is double that of the first LPFG. If both LPFGs are considered separately, the resonance wavelength of the first diffraction order modes in the first LPFG will coincide with the resonance wavelength of the second diffraction order modes of the second LPFG [9]. By adequate parameterization of both LPFGs, interference patterns can be obtained between the attenuation bands of the first diffraction order modes of the first LPFG and the second diffraction order modes of the second LPFG. On the other hand, the first diffraction order modes of the second LPFG will not show interferometry with any modes of the first LPFG.

It is important to note that the cross-coupling coefficient in (2) of each diffraction order mode is directly proportional to the same order component in the Fourier series of the grating profile. For the sake of simplicity, the grating profile is considered rectangular. Consequently, the coefficients are

$$
s_{i}=\frac{w}{\Lambda} \operatorname{sinc}\left(\frac{w}{\Lambda} i\right)
$$

where $w$ is the width of the rectangular wave, $\Lambda$ is the grating period, and $i$ the Fourier order.

If the duty cycle is $50 \%$, the first Fourier series component is much higher than the rest. In this case, the even components of the Fourier series are zero. Therefore, no interference pattern can appear between the first LPFG and the second LPFG with a double period. However, as the duty cycle is increased, 


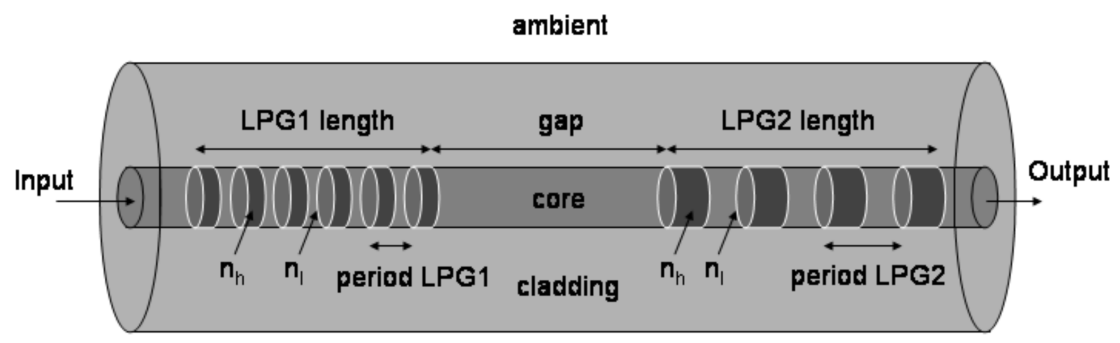

Fig. 1. LPFG structure composed of one LPFG of period $x$ and another LPFG of period $2 x$, separated by a gap.

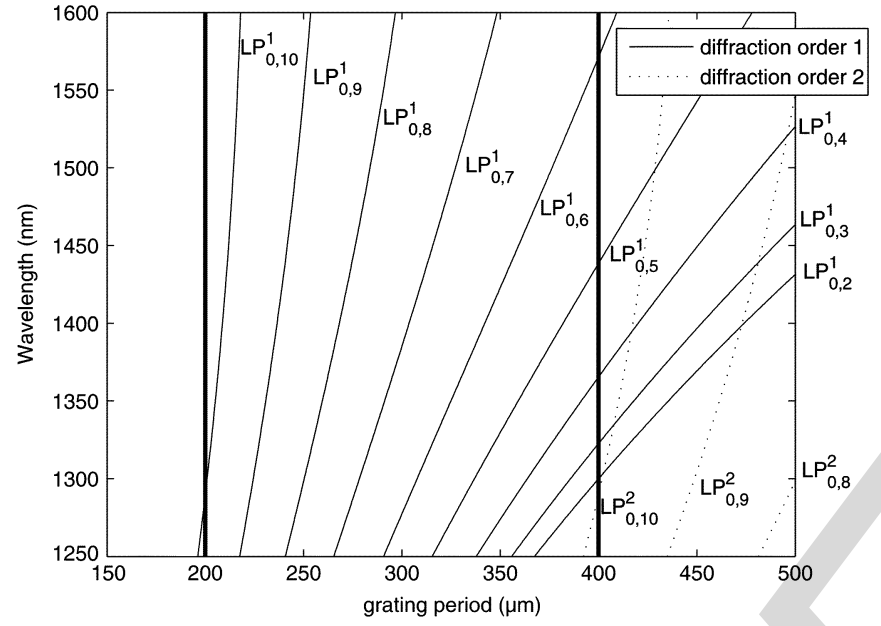

Fig. 2. Theoretical resonance wavelengths as function of grating period.

the second Fourier component is no longer negligible and it approaches the value of the first Fourier component. In this case, interferometric phenomena appear at a cost of a reduction in the absolute value of all the Fourier components except the dc component. At the limit value $100 \%$, all non-dc Fourier components are zero. For the experiments, a duty cycle of $82 \%$ has been considered a good tradeoff value. In this case, the first Fourier component is 0.171 and the second one is 0.144 .

The optical fiber where the two LPFGs were recorded is a Fibercore PS 1250/1500 with a cutoff wavelength of $1155 \mathrm{~nm}$ and a numerical aperture of 0.14 . The LPFGs were written with a point-by-point technique and the source was a $244-\mathrm{nm}$ UV laser beam. The period of the first LPFG was $200 \mu \mathrm{m}$ and the period of the second one was $400 \mu \mathrm{m}$. Light from a broadband source was coupled into the optical fiber and the output signal was recorded in an optical spectrum analyzer (OSA) as the structure was written in the photosensitive optical fiber. The wavelengths analyzed range between 1250 and $1600 \mathrm{~nm}$.

Before showing the experimental results, a previous theoretical analysis was performed with a model based on the LP mode approximation [4], [10]. This scalar model has been used rather than a vectorial method [2] because of the similarity in the results and the improved efficiency that it offers. However, it must be stated that in some circumstances, such as specific overlay deposition, this method is not valid [11]. The notation used henceforth for the modes is $\mathrm{LP}_{0, j}^{k}$, where $j$ is the mode order and $k$ is the diffraction order.

In Fig. 2, some resonance wavelengths are analyzed as a function of the grating period by using (1). The $\mathrm{LP}_{0,10}^{1}$ resonance
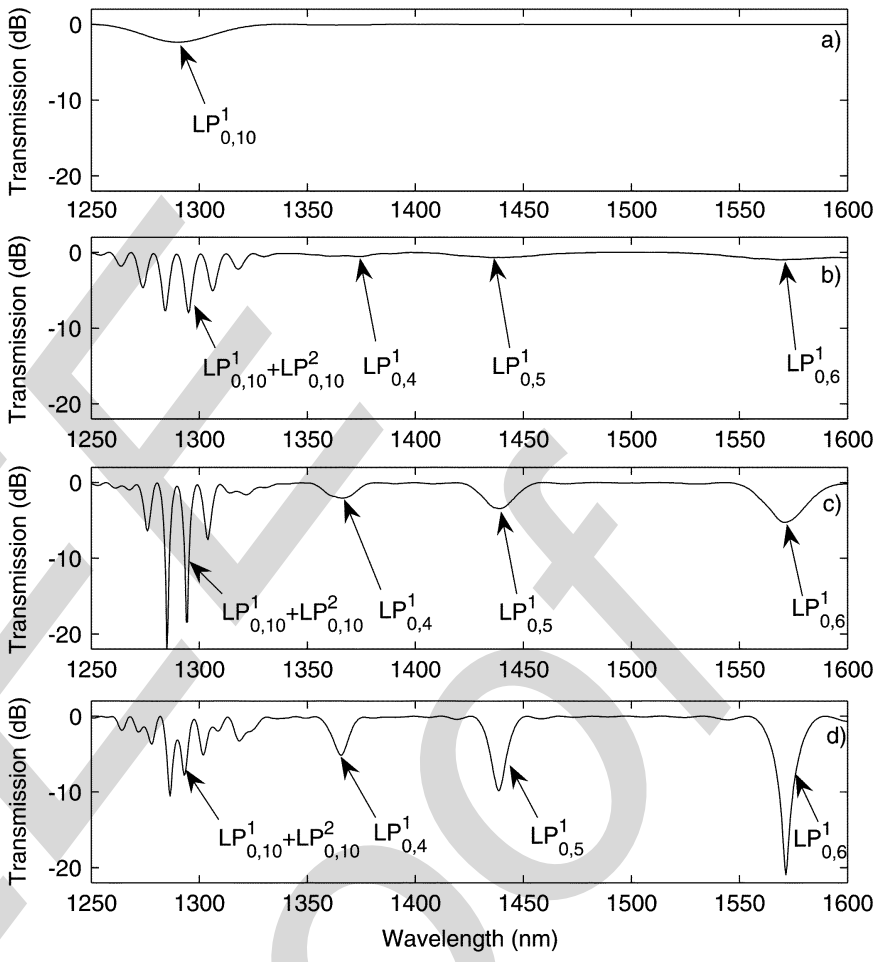

Fig. 3. Theoretical transmission spectra: (a) one $\mathrm{LPFG}$ of $0.95 \mathrm{~cm}$ and period $200 \mu \mathrm{m}$. (b) Two LPFGs of $0.95 \mathrm{~cm}$ and period $200 \mu \mathrm{m}$, and $0.74 \mathrm{~cm}$ and period $400 \mu \mathrm{m}$ separated by $40-\mathrm{mm}$ gap. (c) Two LPFGs of $0.95 \mathrm{~cm}$ and period 200 $\mu \mathrm{m}$, and $1.61 \mathrm{~cm}$ and period $400 \mu \mathrm{m}$ separated by $40-\mathrm{mm}$ gap. (d) Two LPFGs of $0.95 \mathrm{~cm}$ and period $200 \mu \mathrm{m}$, and $2.40 \mathrm{~cm}$ and period $400 \mu \mathrm{m}$ separated by 40-mm gap.

wavelength for a grating period of $200 \mu \mathrm{m}$ (the cross point between the vertical line at $200 \mu \mathrm{m}$ and the $\mathrm{LP}_{0,10}^{1}$ line) coincides with the $\mathrm{LP}_{0,10}^{2}$ resonance wavelength for a grating period $400 \mu \mathrm{m}$. Consequently, both resonances will interfere in the transmission spectrum. On the other hand, for the period 400 $\mu \mathrm{m}$, there are five additional resonances that do not appear for the grating period $200 \mu \mathrm{m}$ : the $\mathrm{LP}_{0,2}^{1}, \mathrm{LP}_{0,3}^{1}, \mathrm{LP}_{0,4}^{1}, \mathrm{LP}_{0,5}^{1}$, and $\mathrm{LP}_{0,6}^{1}$. As a result, these resonances will not present fringes in their respective attenuation bands.

To prove the previous assumptions, the coupled-mode differential equations [4], [10] are used to simulate in Fig. 3 the transmission spectra at different points in the construction of the device of Fig. 1. In order to include the effect of the gap between both fibers, each mode is multiplied by a factor $\exp \left(i \beta z_{0}\right)$, where $\beta$ is the propagation constant of the mode and $z_{0}$ the gap length [2]. The first transmission spectrum presented, Fig. 3(a), considers the simple writing of the first LPFG. The 


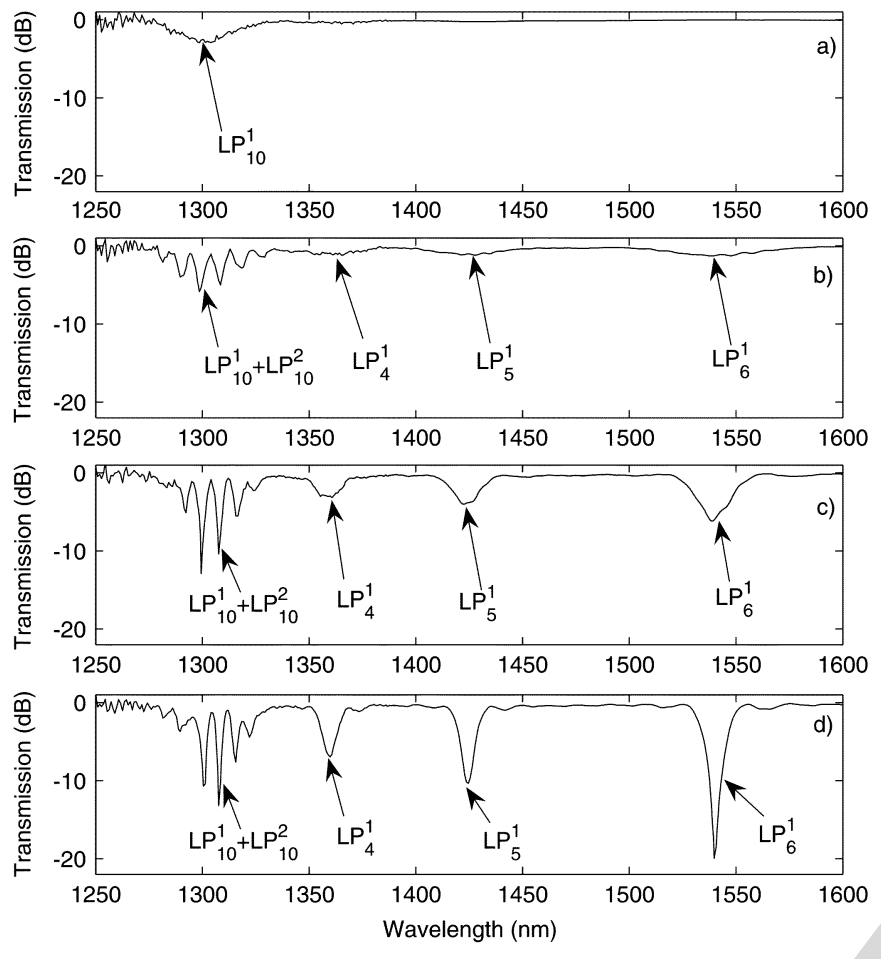

Fig. 4. Experimental transmission spectra for same values of Fig. 3.

length of the LPFG is $0.95 \mathrm{~cm}$, which leads to an attenuation band depth close to $3 \mathrm{~dB}$. This fact permits us to obtain important interferometric fringes. In Fig 3(b), $0.74 \mathrm{~cm}$ of the second LPFG have been also written. Due to the 40-mm gap between both LPFGs, some fringes appear in the attenuation band, and the attenuation bands of the first-order mode resonances of the second LPFG are slightly appreciated: $\mathrm{LP}_{0,4}^{1}, \mathrm{LP}_{0,5}^{1}$, and $\mathrm{LP}_{0,6}^{1}$. In Fig. 3(c), the length of the second LPFG is now $1.61 \mathrm{~cm}$. The fringes are maxima in this case and the attenuation bands of the first-order mode resonances of the second LPFG are clearly appreciated. If we go further to $2.4 \mathrm{~cm}$, the attenuation bands of the first-order mode resonances of the second LPFG are maxima, at a cost of a reduction in the visibility of the fringe pattern of the other attenuation band because there is a recoupling of cladding modes to the core mode. The optimum is the case where the depths of the attenuation bands induced by the first LPFG and the second LPFG are $3 \mathrm{~dB}$. However, even at nonoptimum conditions there is a clear fringe pattern.

The theoretical results of Fig. 3 are corroborated with experimental ones in Fig. 4. The conclusion is that the theory and the experiments agree with the exception of a wavelength shift in the attenuation bands due to some inaccuracies in the parameters that have been used for the simulation.

This device could be used as a sensor. The attenuation band with fringes should be more adequate for the detection of small variations of environmental parameters, because it belongs to a high-order cladding mode and because the narrowband filters show an improved resolution; whereas, the attenuation bands without fringes should be more adequate for higher variations, because they belong to low-order cladding modes and the filters are wider.

\section{CONCLUSION}

In summary, a novel LPFG-based device has been fabricated. Two LPFGs are written in a photosensitive optical fiber with a separation. The periodicity of the second LPFG is double that of the first one. The result is the same interference pattern observed in the attenuation bands of cascaded LPFGs of the same period. In this case, some additional attenuation bands without fringes are created, which belong to the first diffraction order cladding modes of the second LPFG. Consequently, the properties of both types of attenuation bands could be, for instance, combined for the detection of wide and small variations of environmental parameters. The LP mode approximation model has successfully reproduced the experimental results, which indicates the possibility of designing theses devices.

\section{ACKNOWLEDGMENT}

The authors would like to thank M. Andres from the University of Valencia for his kind support and useful comments.

\section{REFERENCES}

[1] A. M. Vengsarkar, P. J. Lemaire, J. B. Judkins, V. Bathia, T. Erdogan, and J. E. Sipe, "Long-period fiber gratings as band rejection filters," $J$. Lightw. Technol., vol. 14, no. 1, pp. 58-65, Jan. 1996.

[2] T. Erdogan, "Fiber grating spectra," J. Lightw. Technol., vol. 15, no. 12, pp. 1277-1294, Dec. 1997.

[3] Y. Koyamada, "Numerical analysis of core-mode to radiation-mode coupling in long-period fiber gratings," IEEE Photon. Technol. Lett., vol. 13, no. 4, pp. 308-310, Apr. 2001.

[4] E. Anemogiannis, E. N. Glytsis, and T. K. Gaylord, "Transmission characteristics of long- period fiber gratings having arbitrary azimutal/ radial refractive index variation," J. Lightw. Technol., vol. 21, no. 3, pp. 218-227, Mar. 2003.

[5] S. W. James and R. P. Tatam, "Optical fibre long-period grating sensors: Characteristics and application," Meas. Sci. Technol., vol. 14, pp. R49-R61, 2003.

[6] B. H. Lee and J. Nishii, "Bending sensitivity of in-series long-period fiber gratings," Opt. Lett., vol. 23, pp. 1624-1626, 1998.

[7] S. W. James, I. Ishaq, G. J. Ashwell, and R. P. Tatam, "Cascaded longperiod gratings with nanostructured coatings," Opt. Lett., vol. 30, pp. 2197-2199, 2005.

[8] D. W. Kim, Y. Zhang, K. L. Cooper, and A. Wang, "In-fiber reflection mode interferometer based on a long-period fiber grating," Appl. Opt., vol. 44, pp. 5368-5373, 2005.

[9] X. Shu, L. Zhang, and I. Bennion, "Fabrication and characterization of ultra-long-period fiber gratings," Opt. Commun., vol. 203, pp. 277-281, 2002.

[10] I. Del Villar, I. R. Matias, F. J. Arregui, and P. Lalanne, "Opitmization of sensitivity in long period fiber gratings," Opt. Expr., vol. 13, pp. $56-69,2005$

[11] I. Del Villar, I. R. Matias, and F. J. Arregui, "Influence in cladding mode distribution of overlay deposition on long-period fiber gratings," J. Opt. Soc. Amer. A, in press. 